International Journal of Applied Mathematical Research, 5(3)(2016) 146-151
International Journal of Applied Mathematical Research
WPC
Website: www.sciencepubco.com/index.php/IJAMR
doi: $10.14419 /$ ijamr.v5i3.6358
Research paper

\title{
Euler-Maruyama approximation of backward doubly stochastic differential delay equations
}

\author{
Sarhan Falah ${ }^{1 *}$ and Jicheng Liu ${ }^{1}$ \\ ${ }^{1}$ School of Mathematics and Statistics, Huazhong University of Science and Technology,, Wuhan, Hubei 430074, P.R.China \\ ${ }^{*}$ Corresponding author E-mail:sarhan_falah@yahoo.com
}

\begin{abstract}
In this paper, we attempt to introduce a new numerical approach to solve backward doubly stochastic differential delay equation ( shortly-BDSDDEs ). In the beginning, we present some assumptions to get the numerical scheme for BDSDDEs, from which we prove important theorem. We use the relationship between backward doubly stochastic differential delay equations and stochastic controls by interpreting BDSDDEs as some stochastic optimal control problems, to solve the approximated BDSDDEs and we prove that the numerical solutions of backward doubly stochastic differential delay equation converge to the true solution under the Lipschitz condition.
\end{abstract}

Keywords: Backward doubly stochastic differential equation; Conditional expectation; Approximation theory; Time delayed coefficients.

\section{Introduction}

Backward stochastic differential equations ( shortly-BSDEs) have been first presented in Pardoux and Peng $[16,17]$ in order to proved existence and uniqueness of the adapted solutions and presented a new class of backward doubly stochastic differential equations, further investigations being (see $[3,4,11,13]$ ). A lot of mathematicians interested in a numerical methods for approximating solution of BSDEs ( see [1, 10, 14, 15, 18, 22]). Xuerong Mao et al. [21] discussed the effects of environmental noise on the delay Lotka-Volterra model. Brahim Boufoussi et al. [2] presented a new class of backward doubly stochastic differential equations, this a new class depend on an integral with respect to an adapted continuous increasing process. Lukasz Delong [5, 6] studied applications of a new class of time-delayed BSDEs and he gives examples of pricing, hedging and portfolio management problems which could be established in the framework of backward stochastic differential delay equation. Wen Lu et al. [19] investigated a class of multivalued backward doubly stochastic differential delay equation, and they proved the existence and uniqueness of the solutions for these equations under Lipschitz condition. Using the Euler-Maruyama method, Xiaotai Wu and Litan Yan [20] defined the numerical solutions of doubly perturbed stochastic delay differential equations driven by Levy process, and they proved the numerical solutions converge to the exact solutions with the local Lipschitz condition. Delong and Imkeller [7] presented a class of BSDEs with time delayed, and they established the existence and uniqueness of a solution for BSDEs with time delayed. Also, they [8] proved the existence and uniqueness as well as the Malliavin's differentiability of the solution for BSDEs with delayed time. Moreover, Diomande and Maticiuc [9] proved the existence and uniqueness of a solution for multivalued BSDEs with time delayed generators. Besides, $\mathrm{Lu}$ and Ren [12] established the existence and uniqueness of the so- lutions for a class of backward doubly stochastic differential equations with time delayed coefficients under Lipschitz condition.

The purpose of this work is to study the numerical convergent of backward doubly stochastic delay differential equations ( shortlyBDSDDEs ) that has the following

$$
\begin{aligned}
Y(t)=\xi & +\int_{t}^{T} f\left(s, Y(s), Z(s), Y_{s}, Z_{s}\right) \mathrm{d} s \\
& +\int_{t}^{T} g\left(s, Y(s), Z(s), Y_{s}, Z_{s}\right) \mathrm{d} B(s)-\int_{t}^{T} Z(s) \mathrm{d} W(s)
\end{aligned}
$$

where $\left\{W_{t}, 0 \leq t \leq T\right\}$ and $\left\{B_{t}, 0 \leq t \leq T\right\}$ are a Brownian motion defined on the probability space $\left(\Omega_{1}, F_{1}, \mathscr{P}_{1}\right)$ and $\left(\Omega_{2}, F_{2}, \mathscr{P}_{2}\right)$, respectively, and $T<\infty$ is a finite time horizon. The coefficients $f$ and $g$ at time $s$ and the terminal condition $\xi$ depend on the past values of a solution $\left(Y_{s}, Z_{s}\right)=(Y(s+\theta), Z(s+\theta))_{-T \leq \theta \leq 0}$.

We point out that the main results in the present paper are different from the Multivalued BDSDEs with time delayed coefficients and Lèvy process established in [19] and [20], respectively. In our work, we extend the approach of BDSDDEs in the general case, and introduce some general assumptions on the numerical convergence of backward doubly stochastic differential equations with time delayed coefficients. Furthermore, we present a numerical scheme based on iterative regression functions which are approximated by projection on vector space of functions. Also, we discuss some theorems about analysis of error. We prove that the approximated solution of BDSDDEs converges to the true solution under Lipschitz condition.

The present paper is organized as follows: In section 2, we present some preliminaries that explain the approximation scheme for BDSDDEs. In section 3, we consider the approximation solution of BDSDDEs and prove some problems that useful for our work. In section 4, we have discussed the numerical convergence under Lipschitz condition. 


\section{Preliminaries and basic assumptions}

In this section, we provide some assumptions and space used in the sequel. Therefore, we consider two independent standard ddimensional Brownian motions $\left\{W_{t}, 0 \leq t \leq T\right\}$ and $\left\{B_{t}, 0 \leq t \leq\right.$ $T\}$, defined on the complete probability spaces $\left(\Omega_{1}, F_{1}, \mathscr{P}_{1}\right)$ and $\left(\Omega_{2}, F_{2}, \mathscr{P}_{2}\right)$, respectively, and a finite time horizon $T<\infty$. We denote

$$
F_{s, t}^{B}=\sigma\left\{B_{r}-B_{s}, s \leq r \leq t\right\}, F_{t}^{W}=\sigma\left\{W_{r}, 0 \leq r \leq t\right\} .
$$

Moreover, we consider $\Omega=\Omega_{1} \times \Omega_{2}, F=F_{1} \otimes F_{2}$ and $\mathscr{P}=\mathscr{P}_{1} \otimes$ $\mathscr{P}_{2}$. In addition, we put

$$
F_{t} \triangleq F_{t}^{W} \otimes F_{s, t}^{B} \otimes \mathscr{N},
$$

where $\mathscr{N}$ is the collection of $\mathscr{P}$-null sets of $F$.That is to say, the $\sigma$-fields $F_{t}, 0 \leq t \leq T$, are $\mathscr{P}$-complete, and the family of $\sigma$-algebras $F=\left\{F_{t}\right\}_{t \in[o, T]}$ is neither increasing nor decreasing, it is not constitute a filtration.

We consider the Euclidian norm $|\cdot|$ in $\mathbb{R}^{k}$ and $\mathbb{R}^{k \times d}$, we use the following spaces

i) Let $L_{-T}^{2}\left(\mathbb{R}^{k \times d}\right)$ is the space of measurable function $Z$ : $[-T, 0] \rightarrow \mathbb{R}^{k \times d}$ such that $\int_{-T}^{0}|Z(t)|^{2} \mathrm{dt}<\infty$.

ii) Let $L_{-T}^{\infty}\left(\mathbb{R}^{k}\right)$ is the space of measurable function $Y:[-T, 0] \rightarrow$ $\mathbb{R}^{k}$ such that $\sup _{-T \leq t \leq 0}|Y(t)|^{2}$ $<\infty$.

iii) Let $H_{T}^{2}\left(\mathbb{R}^{m}\right)$ is the space of $F$-predictable processes $Y: \Omega \times$ $[0, T] \rightarrow \mathbb{R}^{m}$ such that $E \int_{0}^{T}|Y(t)|^{2} \mathrm{dt}<\infty$.

iv) Let $S_{T}^{2}\left(\mathbb{R}^{k}\right)$ is the space of $F$-adapted, product measurable processes $Y: \Omega \times[0, T] \rightarrow \mathbb{R}^{k}$ such that $E\left[\sup _{0 \leq t \leq T}|Y(t)|^{2}\right]<\infty$.

The spaces $H_{T}^{2}\left(\mathbb{R}^{k \times d}\right)$ and $S_{T}^{2}\left(\mathbb{R}^{k}\right)$ are done with the norm $\|Z\|_{H_{T}^{2}}^{2}=$ $E \int_{0}^{T}|Z(t)|^{2} \mathrm{dt}$ and $\|Y\|_{S_{T}^{2}}^{2}=E\left[\sup _{0 \leq t \leq T}|Y(t)|^{2}\right]$, respectively. In this paper, we consider the following BDSDE with time delayed coefficients

$$
\left\{\begin{array}{l}
d(Y(t))=f\left(t, Y(t), Z(t), Y_{t}, Z_{t}\right) d t+g\left(t, Y(t), Z(t), Y_{t}, Z_{t}\right) d B(t) \\
-Z(t) d W(t), 0 \leq t \leq T, \\
Y_{T}=\xi\left(Y_{T}, Z_{T}\right),-T \leq t \leq 0,
\end{array}\right.
$$

where $f$ and $g$ are Borel-measurable functions at time set depend on the past values of the solution $Y_{S}=(Y(s+\theta))_{-T \leq \theta \leq 0}$ and $Z_{S}=$ $(Z(s+\theta))_{-T \leq \theta<0}$. We always set $Z(t)=0$ and $Y(t)=Y(0)$ for $t<0$. Now, we make the following assumptions

Assumption (H1): There exist a positive constant $K_{1}$ and for all $-\tau \leq s<t \leq 0$ such that

$$
E\left[|\xi(t)-\xi(s)|^{2}\right] \leq K_{1}(t-s) .
$$

Assumption (H2): Suppose that $f: \Omega \times[0, T] \times \mathbb{R}^{k} \times \mathbb{R}^{k \times d} \times$ $L_{-T}^{\infty}\left(\mathbb{R}^{k}\right) \times L_{-T}^{2}\left(\mathbb{R}^{k \times d}\right) \rightarrow \mathbb{R}^{k}$ and $g: \Omega \times[0, T] \times L_{-T}^{\infty}\left(\mathbb{R}^{k}\right) \times$ $L_{-T}^{2}\left(\mathbb{R}^{k \times d}\right) \rightarrow \mathbb{R}^{k \times d}$ are product measurable, there exist a positive constants $K_{2}, K_{3}$ and $K_{4}$, and a finite measure $\alpha$ on $[-\tau, 0]$ such that

$$
\begin{gathered}
\left|f\left(t, Y^{1}, Z^{1}, Y_{t}^{1}, Z_{t}^{1}\right)-f\left(t, Y^{2}, Z^{2}, Y_{t}^{2}, Z_{t}^{2}\right)\right|^{2} \leq K_{2}\left(\left|Y^{1}-Y^{2}\right|^{2}+\right. \\
\left.\left|Z^{1}-Z^{2}\right|^{2}\right)+K_{4}\left(\int_{-T}^{0}\left|Y^{1}(t+\theta)-Y^{2}(t+\theta)\right|^{2} \alpha(\mathrm{d} \theta)\right. \\
\left.+\int_{-T}^{0}\left|Z^{1}(t+\theta)-Z^{2}(t+\theta)\right|^{2} \alpha(\mathrm{d} \theta)\right) .
\end{gathered}
$$

and

$$
\begin{gathered}
\left|g\left(t, Y^{1}, Z^{1}, Y_{t}^{1}, Z_{t}^{1}\right)-g\left(t, Y^{2}, Z^{2}, Y_{t}^{2}, Z_{t}^{2}\right)\right|^{2} \leq K_{3}\left(\left|Y^{1}-Y^{2}\right|^{2}+\right. \\
\left.\left|Z^{1}-Z^{2}\right|^{2}\right)+K_{4}\left(\int_{-T}^{0}\left|Y^{1}(t+\theta)-Y^{2}(t+\theta)\right|^{2} \alpha(\mathrm{d} \theta)\right. \\
+\int_{-T}^{0}\left|Z^{1}(t+\theta)-Z^{2}(t+\theta)\right|^{2} \alpha(\mathrm{d} \theta),
\end{gathered}
$$

for all $t \in[0, T],\left(Y^{1}, Z^{1}\right),\left(Y^{2}, Z^{2}\right) \in \mathbb{R}^{k} \times \mathbb{R}^{k \times d},\left(Y_{t}^{1}, Z_{t}^{1}\right),\left(Y_{t}^{2}, Z_{t}^{2}\right) \in$ $L_{-T}^{\infty}\left(\mathbb{R}^{k}\right) \times L_{-T}^{2}\left(\mathbb{R}^{k \times d}\right)$.

Assumption (H3)

$$
E \int_{0}^{T}|f(t, 0,0,0,0)|^{2} \mathrm{dt}<\infty, E \int_{0}^{T}|g(t, 0,0,0,0)|^{2} \mathrm{dt}<\infty .
$$

Assumption(H4)

$$
f(t, \cdot, \cdot, \cdot, \cdot)=0, g(t, \cdot, \cdot)=0
$$

for $t<0$.

Assumption (H5): There exists a positive constant $K_{5}$ such that

$$
|f(Y, Z)|^{2} \vee|g(Y, Z)|^{2} \leq K_{5}\left(1+|Y|^{2}+|Z|^{2}\right),
$$

where $a \vee b=\max \{a, b\}$.

\section{A numerical scheme for BDSDDEs}

In this section, we propose a numerical scheme is based upon a descretization of (1). Moreover, for all integers $n, l \geq 1$ and $t \in$ $[0, T]$, let

$$
-\tau=t_{-l}<t_{-l+1}<\cdots<0=t_{0}<t_{1}<\cdots<t_{n}=T
$$

be a partition of $[-\tau, T]$, and denote

$$
\begin{gathered}
\delta=\triangle t_{i+1}=t_{i+1}-t_{i}=\frac{T}{n}, 1 \leq i \leq n, \triangle B_{t_{i+1}}= \\
B_{t_{i+1}}-B_{t_{i}}, \triangle W_{t_{i+1}}=W_{t_{i+1}}-W_{t_{i}},
\end{gathered}
$$

where $i=0,1, \cdot, \cdot, \cdot, n-1$, and $\triangle t=\max _{-\tau \leq i \leq n-1} \triangle t_{i}$. Now, on the small interval $\left[t_{i}, t_{i+1}\right]$ the equation

$$
\begin{aligned}
Y_{t_{i}}=Y_{t_{i+1}}+\int_{t_{i}}^{t_{i+1}} f\left(s, Y(s), Z(s), Y_{s}, Z_{s}\right) \mathrm{d} s & +\int_{t_{i}}^{t_{i+1}} g\left(s, Y(s), Z(s), Y_{s}, Z_{s}\right) \mathrm{d} B(s) \\
& -\int_{t_{i}}^{t_{i+1}} Z(s) \mathrm{d} W(s)
\end{aligned}
$$

We can be approximated by the discrete equation

$$
\begin{gathered}
Y_{t_{i}}^{n}=Y_{t_{i+1}}^{n}+f\left(t_{i}, Y_{i}^{n}(t), Z_{i}^{n}(t), Y_{i}^{n}(t+\theta), Z_{i}^{n}(t+\theta)\right) \delta+ \\
g\left(t_{i}, Y_{i}^{n}(t), Z_{i}^{n}(t), Y_{i}^{n}(t+\theta), Z_{i}^{n}(t+\theta)\right) \triangle B_{i+1}-Z_{i}^{n}(t) \triangle W_{i+1},
\end{gathered}
$$

with $Y(T)=\xi(T)$ on $-T \leq t \leq 0$. Therefore, we consider a class of BDSDDEs as the form

$$
Y_{i}^{n}(t)=\xi(T)+\int_{0}^{T} f\left(s, Y_{i}^{n}(s), Z_{i}^{n}(s), Y_{i}^{n}(s+\theta), Z_{i}^{n}(s+\theta)\right) \mathrm{d} s+
$$

(2) $\int_{0}^{T} g\left(s, Y_{i}^{n}(s), Z_{i}^{n}(s), Y_{i}^{n}(s+\theta), Z_{i}^{n}(s+\theta)\right) \mathrm{d} B(s)-\int_{0}^{T} Z_{i}^{n}(s) \mathrm{d} W(s)$.

Now, let us define the Euler-Maruyama approximate solution by

$$
\begin{aligned}
& \tilde{Y}(t)=\xi(T)+\int_{0}^{T} f(s, \tilde{Y}(s), \tilde{Z}(s), \tilde{Y}(s+\theta), \tilde{Z}(s+\theta)) \mathrm{d} s \\
& +\int_{0}^{T} g(s, \tilde{Y}(s), \tilde{Z}(s), \tilde{Y}(s+\theta), \tilde{Z}(s+\theta)) \mathrm{d} B(s)-\int_{0}^{T} \tilde{Z}(s) \mathrm{d} W(s) .
\end{aligned}
$$

Now, we present some Lemmas that useful for our work.

Lemma 3.1

Assume the assumptions (H1)-(H2) are fulfilled, then it holds that

$$
E\left[\sup _{0 \leq t \leq T}|Y(t)|^{2}+\int_{0}^{T}|Z(t)|^{2} \mathrm{dt}\right] \leq C M,
$$

where $M=E\left[|\xi|^{2}+\int_{0}^{T}|f(s, o, o, o, o)|^{2} \mathrm{ds}+\int_{0}^{T}|g(s, o, o, o, o)|^{2} \mathrm{ds}\right]$.

Proof. By applying Itố's formula $|Y(t)|^{2}$, we have that

$$
\begin{gathered}
|Y(t)|^{2}+\int_{0}^{T}|Y(s)|^{2} \mathrm{ds}+\int_{0}^{T}|Z(s)|^{2} \mathrm{ds} \leq \\
|\xi|^{2}+2 \int_{0}^{T}\left\langle Y(s), f\left(s, Y(s), Z(s), Y_{s}, Z_{s}\right)\right\rangle \mathrm{ds}+ \\
\int_{0}^{T}\left|g\left(s, Y(s), Z(s), Y_{s}, Z_{s}\right)\right|^{2} \mathrm{ds}+
\end{gathered}
$$

$2 \int_{0}^{T}\left\langle Y(s), g\left(s, Y(s), Z(s), Y_{s}, Z_{s}\right) \mathrm{dB}(\mathrm{s})\right\rangle-2 \int_{0}^{T}\langle Y(s), Z(s) \mathrm{dW}(\mathrm{s})\rangle$,

where $t \in[0, T]$. By using Young's inequality and assumption (H2), we get that 


$$
\begin{gathered}
2 \int_{0}^{T}\left\langle Y(s), f\left(s, Y(s), Z(s), Y_{s}, Z_{s}\right)\right\rangle \mathrm{ds} \leq \\
\gamma \int_{0}^{T}|Y(s)|^{2} \mathrm{ds}+\frac{1}{\gamma} \int_{0}^{T}\left|f\left(s, Y(s), Z(s), Y_{s}, Z_{s}\right)\right|^{2} \mathrm{ds} \leq \\
\gamma \int_{0}^{T}|Y(s)|^{2} \mathrm{ds}+\frac{3}{\gamma} \int_{0}^{T}|f(s, 0,0,0,0)|^{2} \mathrm{ds}+\frac{K_{2}^{2}}{\gamma} \int_{0}^{T}\left(|Y(s)|^{2}+\right. \\
\left.|Z(s)|^{2}\right) \mathrm{ds}+\frac{3 K_{4}}{\gamma} \int_{0}^{T} \int_{-T}^{0}\left(|Y(s+\theta)|^{2}+|Z(s+\theta)|^{2}\right) \alpha(\mathrm{d} \theta) \mathrm{ds}
\end{gathered}
$$
and

$$
\int_{0}^{T}\left|g\left(s, Y(s), Z(s), Y_{s}, Z_{s}\right)\right|^{2} \mathrm{ds} \leq
$$

$6 K_{3}^{2} \int_{0}^{T}\left(|Y(s)|^{2}+|Z(s)|^{2}\right) \mathrm{ds}+3 \int_{0}^{T}|g(s, 0,0,0,0)|^{2} \mathrm{ds}+$

$3 K_{4} \int_{0}^{T} \int_{-T}^{0}\left(|Y(s+\theta)|^{2}+|Z(s+\theta)|^{2}\right) \alpha(\mathrm{d} \theta) \mathrm{ds}$.

By changing of integration order argument, we obtain that

$$
\begin{gathered}
\int_{0}^{T} \int_{-T}^{0}|Y(s+\theta)|^{2} \alpha(\mathrm{d} \theta) \mathrm{ds}=\int_{-T}^{0} \int_{0}^{T}|Y(s+\theta)|^{2} \mathrm{ds} \alpha(\mathrm{d} \theta)= \\
\int_{-T}^{0} \int_{\theta}^{T+\theta}|Y(t)|^{2} \mathrm{dt} \alpha(\mathrm{d} \theta) \leq \beta \int_{0}^{T}|Y(t)|^{2} \mathrm{dt}
\end{gathered}
$$

and

$$
\begin{gathered}
\int_{0}^{T} \int_{-T}^{0}|Z(s+\theta)|^{2} \alpha(\mathrm{d} \theta) \mathrm{ds}=\int_{-T}^{0} \int_{0}^{T}|Z(s+\theta)|^{2} \mathrm{ds} \alpha(\mathrm{d} \theta)= \\
\int_{-T}^{0} \int_{\theta}^{T+\theta}|Z(t)|^{2} \mathrm{dt} \alpha(\mathrm{d} \theta) \leq \beta \int_{0}^{T}|Z(t)|^{2} \mathrm{dt},
\end{gathered}
$$

where $\beta=\int_{-T}^{0} \alpha(\mathrm{d} \theta)$.Therefore, we drive that

$$
\begin{gathered}
|Y(t)|^{2}+\int_{0}^{T}|Y(s)|^{2} \mathrm{ds}+\int_{0}^{T}|Z(s)|^{2} \mathrm{ds} \leq|\xi|^{2}+\gamma \int_{0}^{T}|Y(s)|^{2} \mathrm{ds} \\
+\frac{6 K_{2}^{2}}{\gamma} \int_{0}^{T}\left(|Y(s)|^{2}+|Z(s)|^{2}\right) \mathrm{ds}+\frac{3}{\gamma} \int_{0}^{T}|f(s, 0,0,0,0)|^{2} \mathrm{ds} \\
+3 \int_{0}^{T}|g(s, 0,0,0,0)|^{2} \mathrm{ds}+6 K_{3}^{2} \int_{0}^{T}\left(|Y(s)|^{2}+|Z(s)|^{2}\right) \mathrm{ds} \\
+\left(\frac{3 K_{4} \beta}{\gamma}+3 K_{4} \beta\right) \int_{0}^{T}\left(|Y(s)|^{2}+|Z(s)|^{2}\right) \mathrm{ds} \\
+2 \int_{0}^{T}\left\langle Y(s), g\left(s, Y(s), Z(s), Y_{s}, Z_{S}\right) \mathrm{dB}(\mathrm{s})\right\rangle \\
-2 \int_{0}^{T}\langle Y(s), Z(s) \mathrm{dW}(\mathrm{s})\rangle .
\end{gathered}
$$

By taking the expectation and $t=0$, we obtain that

$$
\begin{gathered}
E|Y(0)|^{2}+C_{1} E \int_{0}^{T}|Y(s)|^{2} \mathrm{ds}+C_{2} E \int_{0}^{T}|Z(s)|^{2} \mathrm{ds} \leq \\
E|\xi|^{2}+\frac{3}{\gamma} E \int_{0}^{T}|f(s, 0,0,0,0)|^{2} \mathrm{ds}+3 E \int_{0}^{T}|g(s, 0,0,0,0)|^{2} \mathrm{ds},
\end{gathered}
$$

where $C_{1}=1-\gamma-\frac{6 K_{2}^{2}}{\gamma}-\frac{3 K_{4} \beta}{\gamma}-3 K_{4} \beta-6 K_{3}^{2}, C_{2}=\varepsilon-\gamma-\frac{6 K_{2}^{2}}{\gamma}-$ $\frac{3 K_{4} \beta}{\gamma}-3 K_{4} \beta-6 K_{3}^{2}, \varepsilon>0$. For sufficiently small $K_{4}$ and $K_{3}$, choosing $\varepsilon>0$ and $\gamma>0$ such that $C_{1}>0$ and $C_{2}>0$, then there exists a constant $C>0$ depending on $\varepsilon, \gamma, K_{2}, K_{3}, K_{4}$ and $\beta$ such that

$$
\begin{gathered}
E \int_{0}^{T}|Y(s)|^{2} \mathrm{ds}+E \int_{0}^{T}|Z(s)|^{2} \mathrm{ds} \leq \\
C\left\{E|\xi|^{2}+E \int_{0}^{T}|f(s, 0,0,0,0)|^{2} \mathrm{ds}+E \int_{0}^{T}|g(s, 0,0,0,0)|^{2} \mathrm{ds}\right\} .
\end{gathered}
$$

Therefore, for $\gamma$ choosing above, we obtain that

$$
\begin{gathered}
\sup _{0 \leq t \leq T}|Y(t)|^{2} \leq \\
|\xi|^{2}+\frac{3}{\gamma} \int_{0}^{T}|f(s, 0,0,0,0)|^{2} \mathrm{ds}+3 \int_{0}^{T}|g(s, 0,0,0,0)|^{2} \mathrm{ds} \\
+2 \sup _{0 \leq t \leq T}\left|\int_{0}^{T}\left\langle Y(s), g\left(s, Y(s), Z(s), Y_{s}, Z_{s}\right) \mathrm{dB}(\mathrm{s})\right\rangle\right| \\
+2 \sup _{0 \leq t \leq T}\left|\int_{0}^{T}\langle Y(s), Z(s) \mathrm{dW}(\mathrm{s})\rangle\right| .
\end{gathered}
$$

Now, by Young's inequality and Burkholder-Davis-Gundy inequality, together with above inequality and assumption $(\mathrm{H} 2)$, there exists a constant $r_{1}>0$ such that

$$
\begin{gathered}
2 E\left[\sup _{0 \leq t \leq T}\left|\int_{0}^{T}\left\langle Y(s), g\left(s, Y(s), Z(s), Y_{s}, Z_{s}\right) \mathrm{dB}(\mathrm{s})\right\rangle\right|\right] \leq \\
r_{1}\left[\lambda_{1} E\left(\sup _{0 \leq t \leq T}|Y(t)|^{2}\right)+\frac{6 K_{3}^{2}+3 K_{4} \beta}{\lambda_{1}} E \int_{0}^{T}|Z(s)|^{2} \mathrm{ds}\right. \\
\left.+\frac{3}{\lambda_{1}} E \int_{0}^{T}|g(s, 0,0,0,0)|^{2} \mathrm{ds}\right] .
\end{gathered}
$$

Similarly, there exists a constant $r_{2}>0$ such that

$$
\begin{gathered}
2 E\left[\sup _{0 \leq t \leq T}\left|\int_{0}^{T}\langle Y(s), Z(s) \mathrm{dW}(\mathrm{s})\rangle\right|\right] \leq \\
r_{2}\left[\lambda_{2} E\left(\sup _{0 \leq t \leq T} \int_{0}^{T}|Y(s)|^{2} \mathrm{ds}\right)+\frac{1}{\lambda_{2}} E\left(\int_{0}^{T}|Z(s)|^{2} \mathrm{ds}\right)\right],
\end{gathered}
$$

where $\lambda_{1}, \lambda_{2}>0$. Then, choosing $\lambda_{1}=\frac{1}{3 r_{1}}$ and $\lambda_{2}=\frac{1}{3 r_{2}}$, for sufficiently small $K_{3}>0$ and $K_{4}>0$, there exists a constant $C>0$ depending on $\varepsilon, \gamma, K_{3}, K_{4}, \beta, r_{1}$ and $r_{2}$ such that

$$
\begin{gathered}
E\left[\sup _{0 \leq t \leq T}|Y(t)|^{2}+\int_{0}^{T}|Z(s)|^{2} \mathrm{ds}\right] \leq \\
C E\left[|\xi|^{2}+\int_{0}^{T}|f(s, 0,0,0,0)|^{2} \mathrm{ds}+\int_{0}^{T}|g(s, 0,0,0,0)|^{2} \mathrm{ds}\right] .
\end{gathered}
$$

Hence, the proof is complete.

\section{Lemma 3.2}

Under assumption (H5), for every $p \geq 2$, there exists a positive constant $M_{1}$, such that

$$
E\left(\sup _{-\tau \leq s \leq T}|Y(s)|^{p}\right) \leq M_{1}
$$

and

$$
E\left(\sup _{-\tau \leq s \leq T}|Z(s)|^{p}\right) \leq M_{2}
$$

Proof. For any $0 \leq s \leq t \leq T$, we get from Eq. (1)

$$
\begin{gathered}
\sup _{0 \leq s \leq T}|Y(s)| \leq \sup _{0 \leq s \leq T} \mid \xi(T)+\int_{0}^{T} f\left(Y(s), Z(S), Y_{S}, Z_{S}\right) \mathrm{ds} \\
+\int_{0}^{T} g\left(Y(s), Z(S), Y_{S}, Z_{S}\right) \mathrm{dB}(\mathrm{s})-\int_{0}^{T} Z(s) \mathrm{dW}(\mathrm{s}) \mid .
\end{gathered}
$$

By taking the expectation, we obtain that

$$
\begin{gathered}
E\left(\sup _{0 \leq s \leq T}|Y(s)|^{p}\right) \leq \\
E\left[\sup _{0 \leq s \leq T} \mid \xi(T)+\int_{0}^{T} f\left(Y(s), Z(S), Y_{s}, Z_{s}\right) \mathrm{ds}\right. \\
\left.+\int_{0}^{T} g\left(Y(s), Z(S), Y_{s}, Z_{s}\right) \mathrm{dB}(\mathrm{s})-\left.\int_{0}^{T} Z(s) \mathrm{dW}(\mathrm{s})\right|^{p}\right] .
\end{gathered}
$$

Thanks to inequality

$$
|a+b+c+d|^{p} \leq 4^{p-1}\left(|a|^{p}+|b|^{p}+|c|^{p}+|d|^{p}\right),
$$

we have that

$$
\begin{gathered}
E\left(\sup _{0 \leq s \leq T}|Y(s)|^{p}\right) \leq 4^{p-1}\left\{E|\xi(T)|^{p}\right. \\
+E\left[\sup _{0 \leq s \leq T}\left|\int_{0}^{T} f\left(Y(s), Z(S), Y_{s}, Z_{s}\right) \mathrm{ds}\right|^{p}\right] \\
+E\left[\sup _{0 \leq s \leq T}\left|\int_{0}^{T} g\left(Y(s), Z(S), Y_{s}, Z_{s}\right) \mathrm{dB}(\mathrm{s})\right|^{p}\right] \\
\left.+E\left[\sup _{0 \leq s \leq T}\left|\int_{0}^{T} Z(s) \mathrm{dW}(\mathrm{s})\right|^{p}\right]\right\} .
\end{gathered}
$$

The Hölder inequality and assumption (H5) imply that

$$
\begin{gathered}
E\left[\sup _{0 \leq s \leq T}\left|\int_{0}^{T} f\left(Y(s), Z(S), Y_{s}, Z_{S}\right) \mathrm{ds}\right|^{p}\right] \leq \\
T^{p-1} E \int_{0}^{T}\left|f\left(Y(s), Z(S), Y_{s}, Z_{s}\right)\right|^{p} \mathrm{ds} \leq \\
T^{p-1} E \int_{0}^{T} K_{1}^{\frac{p}{2}}\left(1+|Y(s)|^{2}+|Z(s)|^{2}+\left|Y_{s}\right|^{2}+\left|Z_{S}\right|^{2}\right)^{\frac{p}{2}} \mathrm{ds} \leq \\
T^{p-1} E \int_{0}^{T} K_{1}^{\frac{p}{2}}\left(1+2 \sup _{0 \leq s \leq T}|Y(s)|^{2}+2 \sup _{0 \leq s \leq T}|Z(s)|^{2}\right)^{\frac{p}{2}} \mathrm{ds} \leq \\
2^{\frac{p-2}{2}} K_{1}^{\frac{p}{2}} T^{p}+2^{p-1} K_{1}^{\frac{p}{2}} T^{p-1} \int_{0}^{T} E\left[\sup _{0 \leq s \leq T}|Y(s)|^{p}\right] \mathrm{ds} \\
+2^{p-1} K_{1}^{\frac{p}{2}} T^{p-1} \int_{0}^{T} E\left[\sup _{0 \leq s \leq T}|Z(s)|^{p}\right] \mathrm{ds} .
\end{gathered}
$$

By Burkholder-Davis-Gundy inequality ( Theorem 1.7.3 in [16]) and assumption (H5), we have that 


$$
\begin{gathered}
E\left[\left.\sup _{0 \leq s \leq T}\left|\int_{0}^{T}\right| g\left(Y(s), Z(S), Y_{s}, Z_{S}\right) \mathrm{dB}(\mathrm{s})\right|^{p}\right] \leq \\
{\left[\frac{p^{p+1}}{2(p-1)^{p-1}}\right]^{\frac{p}{2}} E\left[\int_{0}^{T}\left|g\left(Y(s), Z(S), Y_{s}, Z_{S}\right)\right|^{2} \mathrm{ds}\right]^{\frac{p}{2}} \leq} \\
{\left[\frac{p^{p+1}}{2(p-1)^{p-1}}\right]^{\frac{p}{2}} T^{\frac{p-2}{2}} E\left[\int_{0}^{T}\left|g\left(Y(s), Z(S), Y_{S}, Z_{s}\right)\right|^{p} \mathrm{ds}\right] \leq} \\
+2^{\frac{p-1}{2}} K_{1}^{\frac{p}{2}}\left[\frac{p^{p+1}}{2(p-1)^{p-1}}\right]_{1}^{\frac{p}{2}}\left[\frac{p^{p+1}}{2(p-1)^{p-1}}\right]^{\frac{p}{2}} T^{\frac{p}{2}} \int_{0}^{\frac{p-2}{2}} E\left[\sup _{0 \leq s \leq T}|Y(s)|^{p}\right] \mathrm{ds} \\
+2^{p-1} K_{1}^{\frac{p}{2}}\left[\frac{p^{p+1}}{2(p-1)^{p-1}}\right]^{\frac{p}{2}} T^{\frac{p-2}{2}} \int_{0}^{T} E\left[\sup _{0 \leq s \leq T}|Z(s)|^{p}\right] \mathrm{ds} .
\end{gathered}
$$

Therefore, we have that

$$
E\left[\sup _{0 \leq s \leq T}\left|\int_{0}^{T} Z(s) \mathrm{dW}(\mathrm{s})\right|^{p}\right] \leq \int_{0}^{T} E\left[\sup _{0 \leq s \leq T}|Z(s)|^{p}\right] \mathrm{ds} .
$$

It following from above, we deduce that

$$
\begin{gathered}
E\left(\sup _{0 \leq s \leq T}|Y(s)|^{p}\right) \leq 4^{p-1} E|\xi(T)|^{p}+4^{p-1} 2^{\frac{p-2}{2}} K_{1}^{\frac{p}{2}} T^{p} \\
+4^{p-1} 2^{p-1} K_{1}^{\frac{p}{2}} T^{p-1} \int_{0}^{T} E\left[\sup _{0 \leq s \leq T}|Y(u)|^{p}\right] \mathrm{ds} \\
+4^{p-1} 2^{p-1} K_{1}^{\frac{p}{2}} T^{p-1} \int_{0}^{T} E\left[\sup _{0 \leq s \leq T}|Z(u)|^{p}\right] \mathrm{ds} \\
+4^{p-1} 2^{\frac{p-2}{2}} K_{1}^{\frac{p}{2}}\left[\frac{p^{p+1}}{2(p-1)^{p-1}}\right]^{\frac{p}{2}} T^{\frac{p}{2}} \\
+4^{p-1} 2^{p-1} K_{1}^{\frac{p}{2}}\left[\frac{p^{p+1}}{2(p-1)^{p-1}}\right]^{\frac{p}{2}} T^{\frac{p-2}{2}} \int_{0}^{T} E\left[\sup _{0 \leq s \leq T}|Y(u)|^{p}\right] \mathrm{ds} \\
+4^{p-1} 2^{p-1} K_{1}^{\frac{p}{2}}\left[\frac{p^{p+1}}{2(p-1)^{p-1}}\right]^{\frac{p}{2}} T^{\frac{p-2}{2}} \int_{0}^{T} E\left[\sup _{0 \leq s \leq T}|Z(u)|^{p}\right] \mathrm{ds} \\
+4^{p-1} \int_{0}^{T} E\left[\sup _{0 \leq s \leq T}|Z(s)|^{p}\right] \mathrm{ds} .
\end{gathered}
$$

Now, we assume that

$$
\begin{gathered}
C= \\
\max \left\{4^{p-1}, 4^{p-1} 2^{\frac{p-2}{2}} K_{1}^{\frac{p}{2}} T^{p}, 4^{p-1} 2^{p-1} K_{1}^{\frac{p}{2}} T^{p-1}, 4^{p-1} 2^{p-1} K_{1}^{\frac{p}{2}} T^{p-1}\right. \\
\left., 4^{p-1} 2^{\frac{p-2}{2}} K_{1}^{\frac{p}{2}}\left[\frac{p^{p+1}}{2(p-1)^{p-1}}\right]^{\frac{p}{2}} T^{\frac{p}{2}}, 4^{p-1} 2^{p-1} K_{1}^{\frac{p}{2}}\left[\frac{p^{p+1}}{2(p-1)^{p-1}}\right]^{\frac{p}{2}} T^{\frac{p-2}{2}}\right\} .
\end{gathered}
$$

Therefore, we have that

$$
E\left(\sup _{0 \leq s \leq T}|Y(s)|^{p}\right) \leq C E|\xi(T)|^{P}+C
$$

$+2 C \int_{0}^{T} E\left(\sup _{-\tau \leq u \leq s}|Y(u)|^{p}\right) \mathrm{ds}+C \int_{0}^{T} E\left(\sup _{0 \leq s \leq T}|Z(u)|^{p}\right) \mathrm{ds}$

$+C+C \int_{0}^{T} E\left(\sup _{0 \leq s \leq T}|Z(u)|^{p}\right) \mathrm{ds}+\int_{0}^{T} E\left(\sup _{0 \leq s \leq T}|Z(s)|^{p}\right) \mathrm{ds}$

$$
\begin{aligned}
& \leq C E|\xi(T)|^{p}+2 C+2 C \int_{0}^{T} E\left(\sup _{0 \leq s \leq T}|Y(u)|^{p}\right) \mathrm{ds} \\
& +3 C \int_{0}^{T} E\left(\sup _{0 \leq s \leq T}|Z(s)|^{p}\right) \mathrm{ds} .
\end{aligned}
$$

Notice that

$$
E\left(\sup _{-\tau \leq s \leq T}|Y(s)|^{p}\right) \leq E|| \xi||^{p} \vee E\left(\sup _{0 \leq s \leq T}|Y(s)|^{p}\right),
$$

we have that

$$
\begin{gathered}
E\left(\sup _{-\tau \leq s \leq T}|Y(s)|^{p}\right) \leq 2 C+C E \|\left.\xi(T)\right|^{p} \\
+2 C \int_{0}^{T} E\left(\sup _{0 \leq s \leq T}|Y(u)|^{p}\right) \mathrm{ds}+3 C \int_{0}^{T} E\left(\sup _{0 \leq s \leq T}|Z(s)|^{p}\right) \mathrm{ds} \\
\leq 2 C+C E \| \xi(T)||^{p}+C_{1}\left[\left(\int_{0}^{T} E\left(\sup _{0 \leq s \leq T}|Y(s)|^{2}\right) \mathrm{ds}\right)^{\frac{p}{2}}\right. \\
\left.+\left(\int_{0}^{T} E\left(\sup _{0 \leq s \leq T}|Z(s)|^{2}\right) \mathrm{ds}\right)^{\frac{p}{2}}\right]
\end{gathered}
$$

where $C_{1} \geq 5 C$. From Lemma (3.1), we obtain that

$$
E\left(\sup _{-\tau \leq s \leq T}|Y(s)|^{p}\right) \leq 2 C+C E\|\xi(T)\|^{p}+C_{1} C M,
$$

with $M_{1}=2 C+3 C C_{1} M+2 C E\|\xi\|^{p} e^{C T}$, we have the result, and consequently, we deduce that

$$
E\left(\sup _{-\tau \leq s \leq T}|Z(s)|^{p}\right) \leq M_{2} .
$$

In this point, we consider the following equation on the small interval $\left[t_{i}, t_{i+1}\right]$

$$
\begin{gathered}
Y_{t_{i}}=Y_{t_{i+1}}+\int_{t_{i}}^{t_{i+1}} f\left(s, Y(s), Z(s), Y_{S}, Z_{s}\right) \mathrm{d} s+ \\
\int_{t_{i}}^{t_{i+1}} g\left(s, Y(s), Y_{S}\right) \mathrm{d} B(s)-\int_{t_{i}}^{t_{i+1}} Z(s) \mathrm{d} W(s) .
\end{gathered}
$$

Now, we present the approximating solution as follows

$Z_{t_{i}}=\frac{1}{\triangle t_{i+1}} E\left[\left(Y_{i+1}(t)+g\left(t_{i+1}, Y_{i+1}(t), Y_{i+1}(t+\theta)\right) \triangle B_{i+1}\right) \triangle W_{i+1}\right]$,

and

$$
\begin{aligned}
Y_{t_{i}}=E\left[Y_{i+1}(t)+\right. & \left.g\left(t_{i+1}, Y_{i+1}(t), Y_{i+1}(t+\theta)\right) \triangle B_{i+1}\right] \\
& +f\left(t_{i}, Y_{i}(t), Z_{i}(t), Y_{i}(t+\theta), Z_{i}(t+\theta)\right) \triangle t_{i+1} .
\end{aligned}
$$

\section{Representation results for BDSDDEs}

This section is devoted to the study of the approximated solution of BDSDDEs converges to the exact solution under Lipschitz condition. The main result of this section is the following

Theorem 4.1

Suppose that assumption (H2) is fulfilled. For each $1 \leq i \leq n$, then it holds that

$$
\begin{gathered}
\lim _{\triangle t_{i+1} \rightarrow 0} E\left[\max _{1 \leq i \leq n} \sup _{t_{i} \leq t \leq t_{i+1}}\left|Y_{i}(t)-\tilde{Y}_{i}(t)\right|^{2}\right. \\
\left.+\sum_{i=1}^{n} \int_{t_{i}}^{t_{i+1}}\left|Z_{i}(s)-\tilde{Z}_{i}(s)\right|^{2} \mathrm{ds}\right]<\infty
\end{gathered}
$$

where $E\left[\max _{1 \leq i \leq n} \sup _{t_{i} \leq t \leq t_{i+1}}\left|Y_{i+1}(t)-\tilde{Y}_{i+1}(t)\right|^{2}\right]<\infty$.

Proof. In the beginning, we take the first term $E\left[\max _{1 \leq i \leq n} \sup _{t_{i} \leq t \leq t_{i+1}}\left|Y_{i}(t)-\tilde{Y}_{i}(t)\right|^{2}\right], \quad$ and from equation (6)

$$
\begin{gathered}
\left|Y_{i}(t)-\tilde{Y}_{i}(t)\right|=\mid E\left[Y_{i+1}(t)-\tilde{Y}_{i+1}(t)\right. \\
+\left(g\left(t_{i+1}, Y_{i+1}(t), Y_{i+1}(t+\theta)\right)\right. \\
\left.\left.-g\left(t_{i+1}, \tilde{Y}_{i+1}(t), \tilde{Y}_{i+1}(t+\theta)\right)\right) \triangle B_{i+1}\right] \\
+\left(f\left(t_{i}, Y_{i}(t), Z_{i}(t), Y_{i}(t+\theta), Z_{i}(t+\theta)\right)\right. \\
\left.-f\left(t_{i}, \tilde{Y}_{i}(t), \tilde{Z}_{i}(t), \tilde{Y}_{i}(t+\theta), \tilde{Z}_{i}(t+\theta)\right)\right) \triangle_{i+1} \mid .
\end{gathered}
$$

Using elementary inequality $|a+b|^{2} \leq 2\left(|a|^{2}+|b|^{2}\right)$, we have that

$$
\begin{gathered}
\left|Y_{i}(t)-\tilde{Y}_{i}(t)\right|^{2} \leq 2 E \mid Y_{i+1}(t)-\tilde{Y}_{i+1}(t) \\
+\left(g\left(t_{i+1}, Y_{i+1}(t), Y_{i+1}(t+\theta)\right)\right. \\
\left.-g\left(t_{i+1}, \tilde{Y}_{i+1}(t), \tilde{Y}_{i+1}(t+\theta)\right)\right)\left.\triangle B_{i+1}\right|^{2} \\
+2 \mid\left(f\left(t_{i}, Y_{i}(t), Z_{i}(t), Y_{i}(t+\theta), Z_{i}(t+\theta)\right)\right. \\
\left.-f\left(t_{i}, \tilde{Y}_{i}(t), \tilde{Z}_{i}(t), \tilde{Y}_{i}(t+\theta), \tilde{Z}_{i}(t+\theta)\right)\right)\left.\triangle \tilde{Y}_{i+1}\right|^{2} \leq \\
4 E\left|Y_{i+1}(t)-\tilde{Y}_{i+1}(t)\right|^{2}+4 E \mid\left(g\left(t_{i+1}, Y_{i+1}(t), Y_{i+1}(t+\theta)\right)\right. \\
-\left.g\left(t_{i+1}, \tilde{Y}_{i+1}(t), \tilde{Y}_{i+1}(t+\theta)\right) \triangle B_{i+1}\right|^{2} \\
+2 \triangle \triangle_{i+1}^{2} \mid f\left(t_{i}, Y_{i}(t), Z_{i}(t), Y_{i}(t+\theta), Z_{i}(t+\theta)\right) \\
-\left.f\left(t_{i}, \tilde{Y}_{i}(t), \tilde{Z}_{i}(t), \tilde{Y}_{i}(t+\theta), \tilde{Z}_{i}(t+\theta)\right)\right|^{2} .
\end{gathered}
$$

By taking the expectation, we get that 


$$
\begin{gathered}
E\left|Y_{i}(t)-\tilde{Y}_{i}(t)\right|^{2} \leq 2 E\left[E\left|Y_{i+1}(t)-\tilde{Y}_{i+1}(t)\right|^{2}\right] \\
+4 E\left[E \mid\left(g\left(t_{i+1}, Y_{i+1}(t), Y_{i+1}(t+\theta)\right)\right.\right. \\
\left.\left.-g\left(t_{i+1}, \tilde{Y}_{i+1}(t), \tilde{Y}_{i+1}(t+\theta)\right)\right)\left.\right|^{2}\right] E\left|\triangle B_{i+1}\right|^{2} \\
+2 \triangle_{i+1}^{2} E \mid f\left(t_{i}, Y_{i}(t), Z_{i}(t), Y_{i}(t+\theta), Z_{i}(t+\theta)\right) \\
-\left.f\left(t_{i}, \tilde{Y}_{i}(t), \tilde{Z}_{i}(t), \tilde{Y}_{i}(t+\theta), \tilde{Z}_{i}(t+\theta)\right)\right|^{2}
\end{gathered}
$$

By using assumption (H2), we have that

$$
\begin{gathered}
E\left|Y_{i}(t)-\tilde{Y}_{i}(t)\right|^{2} \leq 2 E\left|Y_{i+1}(t)-\tilde{Y}_{i+1}(t)\right|^{2} \\
+4 \triangle_{i+1} E\left(K_{3}\left|Y_{i+1}(t)-\tilde{Y}_{i+1}(t)\right|^{2}\right. \\
\left.+K_{4} \int_{-T}^{0}\left|Y_{i+1}(t+\theta)-\tilde{Y}_{i+1}(t+\theta)\right|^{2} \alpha(\mathrm{d} \theta)\right) \\
+2 \triangle_{i+1}^{2} E\left[K_{2}\left(\left|Y_{i}(t)-\tilde{Y}_{i}(t)\right|^{2}+\left|Z_{i}(t)-\tilde{Z}_{i}(t)\right|^{2}\right)\right. \\
+K_{4}\left(\int_{-T}^{0}\left|Y_{i}(t+\theta)-\tilde{Y}_{i}(t+\theta)\right|^{2} \alpha(\mathrm{d} \theta)\right. \\
\left.\left.+\int_{-T}^{0}\left|Z_{i+1}(t+\theta)-\tilde{Z}_{i+1}(t+\theta)\right|^{2} \alpha(\mathrm{d} \theta)\right)\right] .
\end{gathered}
$$

Therefore, we have that

$$
\begin{gathered}
E\left|Y_{i}(t)-\tilde{Y}_{i}(t)\right|^{2}-2 \triangle_{i+1}^{2} K_{2} E\left|Y_{i}(t)-\tilde{Y}_{i}(t)\right|^{2} \leq \\
2 E\left|Y_{i+1}(t)-\tilde{Y}_{i+1}(t)\right|^{2}+4 \triangle_{i+1} K_{3} E\left|Y_{i+1}(t)-\tilde{Y}_{i+1}(t)\right|^{2} \\
+4 \triangle_{i+1} K_{4} E \int_{-T}^{0}\left|Y_{i+1}(t+\theta)-\tilde{Y}_{i+1}(t+\theta)\right|^{2} \alpha(\mathrm{d} \theta) \\
\quad+2 \triangle_{i+1}^{2} K_{2} E\left|Z_{i}(t)-\tilde{Z}_{i}(t)\right|^{2} \\
\quad+2 \triangle_{i+1}^{2} K_{4} E \int_{-T}^{0}\left|Y_{i}(t+\theta)-\tilde{Y}_{i}(t+\theta)\right|^{2} \alpha(\mathrm{d} \theta) \\
+2 \triangle_{i+1}^{2} K_{4} E \int_{-T}^{0}\left|Z_{i}(t+\theta)-\tilde{Z}_{i}(t+\theta)\right|^{2} \alpha(\mathrm{d} \theta) .
\end{gathered}
$$

And then, we get that

$$
\begin{gathered}
\left(1-2 \triangle_{i+1}^{2}\right) E\left|Y_{i}(t)-\tilde{Y}_{i}(t)\right|^{2} \leq\left(2+4 \triangle_{i+1} K_{3}\right) E\left|Y_{i+1}(t)-\tilde{Y}_{i+1}(t)\right|^{2} \\
+2 \triangle_{i+1}^{2} K_{2} E\left|Z_{i}(t)-\tilde{Z}_{i}(t)\right|^{2} \\
+\left(4 \triangle_{i+1} K_{4}+2 \triangle_{i+1}^{2} K_{4}\right) E \int_{-T}^{0}\left|Y_{i}(t+\theta)-\tilde{Y}_{i}(t+\theta)\right|^{2} \alpha(\mathrm{d} \theta) \\
+2 \triangle_{i+1}^{2} K_{4} E \int_{-T}^{0}\left|Z_{i}(t+\theta)-\tilde{Z}_{i}(t+\theta)\right|^{2} \alpha(\mathrm{d} \theta) .
\end{gathered}
$$

Now, we take the following

$$
\begin{gathered}
\left|Z_{t_{i}}-\tilde{Z}_{t_{i}}\right| \leq \mid \frac{1}{\triangle_{i+1}} E\left[\left(\left(Y_{i+1}(t)-\tilde{Y}_{i+1}(t)\right)\right.\right. \\
+\left(g \left(t_{i+1}, Y_{i+1}(t), Y_{i+1}(t+\theta)\right.\right. \\
\left.\left.\left.-g\left(t_{i+1}, \tilde{Y}_{i+1}(t), \tilde{Y}_{i+1}(t+\theta)\right)\right) \triangle B_{i+1}\right) \triangle W_{i+1}\right] \mid .
\end{gathered}
$$

Using elementary inequality $|a+b|^{2} \leq 2\left(|a|^{2}+|b|^{2}\right)$, we get that

$$
\begin{gathered}
\left|Z_{i}(t)-\tilde{Z}_{i}(t)\right|^{2} \leq \frac{2}{\triangle_{i+1}^{2}} E\left|Y_{i+1}(t)-\tilde{Y}_{i+1}(t)\right|^{2} E\left|\triangle W_{i+1}\right|^{2} \\
\quad+\frac{2}{\triangle_{i+1}^{2}} E \mid g\left(t_{i+1}, Y_{i+1}(t), Y_{i+1}(t+\theta)\right) \\
-\left.g\left(t_{i+1}, \tilde{Y}_{i+1}(t), \tilde{Y}_{i+1}(t+\theta)\right)\right|^{2} E\left|\triangle B_{i+1}\right|^{2} E\left|\triangle W_{i+1}\right|^{2} .
\end{gathered}
$$

By taking the expectation, we have that

$$
\begin{gathered}
\left.E\left|Z_{i}(t)-\tilde{Z}_{i}(t)\right|^{2} \leq \frac{2}{\triangle_{i+1}} E \mid Y_{i+1}(t)-\tilde{Y}_{i+1}(t)\right)\left.\right|^{2} \\
+2 E\left|g\left(t_{i+1}, Y_{i+1}(t), Y_{i+1}(t+\theta)\right)-g\left(t_{i+1}, \tilde{Y}_{i+1}(t), \tilde{Y}_{i+1}(t+\theta)\right)\right|^{2} .
\end{gathered}
$$

By using assumption (H2), we deduce that

$$
\begin{gathered}
\left.E\left|Z_{i}(t)-\tilde{Z}_{i}(t)\right|^{2} \leq \frac{2}{\triangle_{i+1}} E \mid Y_{i+1}(t)-\tilde{Y}_{i+1}(t)\right)\left.\right|^{2}+2 E\left(K_{3} \mid Y_{i+1}(t)\right. \\
\left.-\left.\tilde{Y}_{i+1}(t)\right|^{2}\right)+2 K_{4} E \int_{-T}^{0}\left|Y_{i+1}(t+\theta)-\tilde{Y}_{i+1}(t+\theta)\right|^{2} \alpha(\mathrm{d} \theta) .
\end{gathered}
$$

From above inequality, we have that

$$
\begin{gathered}
\left.\left(1-2 \triangle_{i+1}^{2}\right) E\left|Y_{t_{i}}-\tilde{Y}_{t_{i}}\right|^{2} \leq\left(2+4 \triangle_{i+1} K_{3}\right) E \mid Y_{i+1}(t)-\tilde{Y}_{i+1}(t)\right)\left.\right|^{2} \\
+2 \triangle_{i+1}^{2} K_{2}\left[\frac{2}{\triangle_{i+1}} E\left|Y_{i+1}(t)-\tilde{Y}_{i+1}(t)\right|^{2}+2 E\left(K_{3} \mid Y_{i+1}(t)\right.\right. \\
\left.\left.-\left.\tilde{Y}_{i+1}(t)\right|^{2}\right)+2 K_{4} E \int_{-T}^{0}\left|Y_{i+1}(t+\theta)-\tilde{Y}_{i+1}(t+\theta)\right|^{2} \alpha(\mathrm{d} \theta)\right] \\
+\left(4 \triangle_{i+1} K_{4}+2 \triangle_{i+1}^{2} K_{4}\right) E \int_{-T}^{0}\left|Y_{i}(t+\theta)-\tilde{Y}_{i}(t+\theta)\right|^{2} \alpha(\mathrm{d} \theta) \\
+2 \triangle_{i+1}^{2} K_{4} E \int_{-T}^{0}\left|Z_{i}(t+\theta)-\tilde{Z}_{i}(t+\theta)\right|^{2} \alpha(\mathrm{d} \theta)
\end{gathered}
$$

Therefore, we have that

$$
\begin{gathered}
E\left|Y_{t_{i}}-\tilde{Y}_{t_{i}}\right|^{2} \leq \frac{2+4 \triangle_{i+1} K_{3}+4 \triangle_{i+1} K_{2}+2 K_{3}}{1-2 \triangle_{i+1}^{2}} E\left|Y_{i+1}(t)-\tilde{Y}_{i+1}(t)\right|^{2} \\
+\frac{4 \triangle_{i+1}^{2} K_{2} K_{4}+4 \triangle_{i+1} K_{4}+2 \triangle_{i+1}^{2} K_{4}}{1-2 \triangle_{i+1}^{2}} E \int_{-T}^{0} \mid Y_{i+1}(t+\theta) \\
-\left.\tilde{Y}_{i+1}(t+\theta)\right|^{2} \alpha(\mathrm{d} \theta)+\frac{4 \triangle_{i+1} K_{4}+2 \triangle_{i+1}^{2} K_{4}}{1-2 \triangle_{i+1}^{2}} E \int_{-T}^{0} \mid Y_{i}(t+\theta) \\
-\left.\tilde{Y}_{i}(t+\theta)\right|^{2} \alpha(\mathrm{d} \theta)+\frac{2 \triangle_{i+1}^{2} K_{4}}{1-2 \triangle_{i+1}^{2}} E \int_{-T}^{0} \mid Z_{i+1}(t+\theta) \\
-\left.\tilde{Z}_{i+1}(t+\theta)\right|^{2} \alpha(\mathrm{d} \theta) .
\end{gathered}
$$

By taking the Limit, we get that

$$
\lim _{\triangle_{i+1} \rightarrow 0} E\left|Y_{t_{i}}-\tilde{Y}_{t_{i}}\right|^{2} \leq\left(2+2 K_{3}\right) E\left|Y_{i+1}(t)-\tilde{Y}_{i+1}(t)\right|^{2} .
$$

Therefore, we have that

$$
\begin{gathered}
\lim _{\triangle_{i+1} \rightarrow 0} E\left[\max _{1 \leq i \leq n} \sup _{t_{i} \leq t \leq t_{i+1}}\left|Y_{i}(t)-\tilde{Y}_{i}(t)\right|^{2}\right] \leq \\
\left(2+2 K_{3}\right) E\left[\max _{1 \leq i \leq n} \sup _{t_{i} \leq t \leq t_{i+1}}\left|Y_{i+1}(t)-\tilde{Y}_{i+1}(t)\right|^{2}\right]<\infty .
\end{gathered}
$$

By the same way above, we deduce that

$$
\lim _{\triangle_{i+1} \rightarrow 0} E\left[\sum_{i=1}^{n} \int_{t_{i}}^{t_{i+1}}\left|Z_{i}(s)-\tilde{Z}_{i}(s)\right|^{2} \mathrm{ds}\right]<\infty .
$$

\section{Remark 4.2}

Suppose that assumption (H2)-(H5) are fulfilled, we deduce that

$$
\begin{gathered}
\lim _{\triangle t_{i+1} \rightarrow 0} E\left[\max _{1 \leq i \leq n} \sup _{t_{i} \leq t \leq t_{i+1}}\left|Y_{i}(t)-\tilde{Y}_{i}(t)\right|^{p}\right. \\
\left.\quad+\sum_{i=1}^{n} \int_{t_{i}}^{t_{i+1}}\left|Z_{i}(s)-\tilde{Z}_{i}(s)\right|^{p} \mathrm{ds}\right]^{\frac{1}{p}}<\infty
\end{gathered}
$$

for all $p \geq 2$ and $1 \leq i \leq n$.

Theorem 4.3

Suppose that assumption (H1)-(H5) are fulfilled.If the Lipschitz coefficients $K_{2}, K_{3}$ and $K_{4}$ are small enough, then there exists a unique solution of (2).

Proof. Existence. By Theorem 4.1, then there exist $Y \in S_{T}^{2}\left(\mathbb{R}^{k}\right)$ and $Z \in H_{T}^{2}\left(\mathbb{R}^{k \times d}\right)$ such that

$$
\lim _{n \rightarrow \infty}\left(Y^{n}, Z^{n}\right)=(Y, Z) .
$$

Then Lemma 3.2 and Theorem 4.1 show that

$$
\lim _{n \rightarrow \infty}\left(Y^{n}\right)
$$

in $S_{T}^{2}\left(\mathbb{R}^{k}\right)$ and

$$
\lim _{n \rightarrow \infty} E\left|Y^{n}(t)-Y(t)\right|^{2}=0,0 \leq t \leq T .
$$

From Fatou's Lemma, Lemma 3.1, Lemma 3.2 and Theorem 4.1, we get the result.

Uniqueness. Let $\left.Y^{i}(t), Z^{i}(t)\right), i=1,2$ be two solutions of BDSDDE (2). By Itô's formula, we deduce that

$$
\begin{gathered}
\left|Y^{1}(t)-Y^{2}(t)\right|^{2}+\int_{t}^{T}\left|Y^{1}(s)-Y^{2}(s)\right|^{2} \mathrm{ds}+\int_{t}^{T}\left|Z^{1}(s)-Z^{2}(s)\right|^{2} \mathrm{ds} \leq \\
2 \int_{t}^{T}\left\langle Y^{1}(s)-Y^{2}(s),\left(f\left(s, Y^{1}(s), Z^{1}(s), Y^{1}(s+\theta), Z^{1}(s+\theta)\right)\right.\right. \\
\left.\left.-f\left(s, Y^{2}(s), Z^{2}(s), Y^{2}(s+\theta), Z^{2}(s+\theta)\right)\right)\right\rangle \mathrm{ds} \\
+\int_{t}^{T} \mid f\left(s, Y^{1}(s), Z^{1}(s), Y^{1}(s+\theta), Z^{1}(s+\theta)\right) \\
-\left.f\left(s, Y^{2}(s), Z^{2}(s), Y^{2}(s+\theta), Z^{2}(s+\theta)\right)\right|^{2} \mathrm{ds} \\
+2 \int_{t}^{T}\left\langle\left\langle Y^{1}(t)-Y^{2}(t),\left(g\left(s, Y^{1}(s), Z^{1}(s), Y^{1}(s+\theta), Z^{1}(s+\theta)\right)\right.\right.\right. \\
\left.\left.-g\left(s, Y^{2}(s), Z^{2}(s), Y^{2}(s+\theta), Z^{2}(s+\theta)\right)\right) \mathrm{dB}(\mathrm{s})\right\rangle \\
-2 \int_{t}^{T}\left\langle Y^{1}(s)-Y^{2}(s),\left(Z^{1}(s)-Z^{2}(s)\right) \mathrm{dW}(\mathrm{s})\right\rangle .
\end{gathered}
$$

As the same proof of Lemma 3.1, we have the result. 


\section{References}

[1] V. Bally, Approximation scheme for solution of BSDE, Backward stochastic Differential Equations (N.EI Karoui and L. Mazzliak, eds.), Pitman, London, 1997, 177-191.

[2] B. Brahim, V.C. Jan and N. Mrhardy, Generalized backward doubly stochastic differential equations and SPDEs with nonlinear numerical boundary conditions, Bernoulli, 2007, Vol. 13, No. 2, 433-446.

[3] Gh. Constantin, Uniqueness for backward stochastic differential equations with non-Lipschiz coefficionts, Anal. Univ. Timisoara Ser. MatInform. XXXIX (2001) 37-43.

[4] Gh. Constantin, On the existence and uniqueness of adapted solutions for backward stochastic differential equations, Anal. Univ. Timisoara Ser. Mat-Inform. XXXIX (2001) 15-22.

[5] L. Delong, Applications of time-delayed backward stochastic differential equations to pricing, hedging and management of insurance and financial risks. Preprint. 2010, arxiv: 1005. 4417.

[6] L. Delong, Applications of time-delayed backward stochastic differential equations to pricing, hedging and portfolio meanagement, 2011, Al. Niepodleglosei 162, 02-554 warsaw, poland.

[7] L. Delong and P. Imkeller, Backward stochastic differential equations with time delayed generators-results and counterexamples. Ann. Appl. probab. 20(2010) 1512-1536.

[8] L. Delong and P. Imkeller, On Malliavin's differentiability of BSDE with time delayed generators driven by Brownian motions and Poisson random measures. Stochastic process. Appl. 120 (2010) 1748-1775.

[9] B. Diomande and L. Maticiuc, Multivalued backward stochastic differential equations with time delayed generators. preprint. 2013, arxiv: $1305.7170 \mathrm{v} 1$

[10] J. Douglas and J. Ma, P. Protter, Numerical methods for forwardbackward stochastic differential equations, Ann. Appl. Probab., 6, 1996, 940-968

[11] J. P. Lepeltier and J. San Martin, Backward stochastic differential equations with continuous generator, statist. Probab. Letters, 32, 1997 425-430.

[12] W. Lu and Y. Ren, Backward doubly stochastic differential equations with time delayed coefficients. submitted, 2012.

[13] X. Mao, Adapted solutions of backward stochastic differential equations with non-Lipschiz coefficients, Stoch. Proc. and their Appli 58 , 1995, 281-292.

[14] J. Ma, P. Protter and J. Yong, Solving forward-backward stochastic differential equations explicitly- a four step scheme, Probab. Theory Related Fields, 98, 1994, 339-359.

[15] J. Ma, Ph. Protter, J. San Martin and S. Torres, Numerical Method for Backward stochastic Differential Equations, The Annals of Applied Probability, 2002, Vol. 12, no. 1, 302-316.

[16] E. Pardoux and S. Peng, Adapted Solution of a backward stochastic differential equation, systems and control Letters, 14,1990, 55-61.

[17] E. pardoux and S. peng, Backward doubly stochastic differential equations and systems of quasilinear SPDEs probab. Theory Related Fields, 88 (1994) 209-227.

[18] Y. Wang and Z. Huang, Backward stochastic differential equations with non-Lipschitz coefficients, stat. and Prob. Letters 79, 2009, 1438 1443

[19] L. Wen, R. Yong and H. Lanying, Multivalued bachward doubly stochastic differential equations with time delayed coefficients, 13 Aug 2013 arxiv:1308.2748v1 [math. PR].

[20] W. Xiaotai and Y. Litan, Numerical solutions of doubly perturbed stochastic delay differential equations driven by Levy process, Arab J Math (2012) 1: 251-265.

[21] M. Xuerong, Y. chenggui and Z. Jiezhong, Stochastic differential delay equations of population dynamics, J. Math. Anal. Appl. 304 (2005) 296-320.

[22] J. Zhang, A numerical scheme for BSDEs. The Annaals of Applied Probability, (2004) vol. 14; No 1, 459-488. 